\title{
Ultrafast optical response of plasmonic structures beyond the perturbative regime: evidence of universal saturation dynamics
}

\author{
Mychel Gonçalves Silva ${ }^{1 *}$, Danielle Cristina Teles-Ferreira ${ }^{1}$, Cristian Manzoni ${ }^{2}$, Lucia \\ Ganzer $^{3}$, Livia Siman ${ }^{1}$, Claudilene Ribeiro Chaves ${ }^{4}$, Luiz Orlando Ladeira ${ }^{1}$, Giulio \\ Cerullo $^{2,3, *}$, Stefano Longhi ${ }^{2,3}$, Sandro De Silvestri ${ }^{2,3}$, Giuseppe Della Valle ${ }^{2,3, *}$, and Ana \\ Maria de Paula, \\ ${ }^{1}$ Departamento de Física, Universidade Federal de Minas Gerais, 31270-901 Belo Horizonte-MG, \\ Brazil \\ ${ }^{2}$ Istituto di Fotonica e Nanotecnologie, Consiglio Nazionale delle Ricerche, Piazza L. da Vinci 32, I- \\ 20133 Milano, Italy \\ ${ }^{3}$ Dipartimento di Fisica, Politecnico di Milano, Piazza L. da Vinci 32, I-20133 Milano, Italy \\ ${ }^{4}$ Departamento de Biointeração-ICS, Universidade Federal da Bahia, 40110-100 Salvador-BA, Brazil
}

\begin{abstract}
The transient optical response of gold nanorods is investigated beyond the perturbative regime. Ultrafast pump-probe spectroscopy and semiclassical modeling of hot electrons reveal a universal mechanism presiding over the saturation of nonlinear plasmonic effects.
\end{abstract}

Plasmon dynamics in metal nanomaterials has been the subject of intensive research, mostly because of the potential applications (from plasmon-enhanced photovoltaics to ultrafast nanophotonics) and for the beauty of the underlying physical phenomena presiding over the observed optical features. However, most of research dealt with the perturbative regime of the transient optical response. In this work we report an experimental and theoretical study of the transient optical response of plasmonic nanostructures beyond the perturbative regime. Using gold nanorods dispersed in water as a model system, we combine ultrafast pump-probe spectroscopy and semiclassical modeling of hot electron dynamics to investigate the dependence of the plasmon transient response on the pump fluence.

The pump-probe experiments are performed with an amplified Ti:Sapphire system generating $100 \mathrm{fs}$ pulses at $800 \mathrm{~nm}$ wavelength and $1 \mathrm{kHz}$ repetition rate. The pump is either the fundamental wavelength or the second harmonic at $400 \mathrm{~nm}$ generated in a BBO crystal. The probe is obtained by passing the light through a sapphire crystal to generate a broadband white light continuum covering a spectral range from $430 \mathrm{~nm}$ to $700 \mathrm{~nm}$. The differential transmission $(\Delta \mathrm{T} / \mathrm{T})$ spectrum is detected with an optical multichannel analyser.

* Corresponding authors: mychelgs@gmail.com , ana@fisica.ufmg.br , giulio.cerullo@polimi.it, giuseppe.dellavalle@polimi.it 


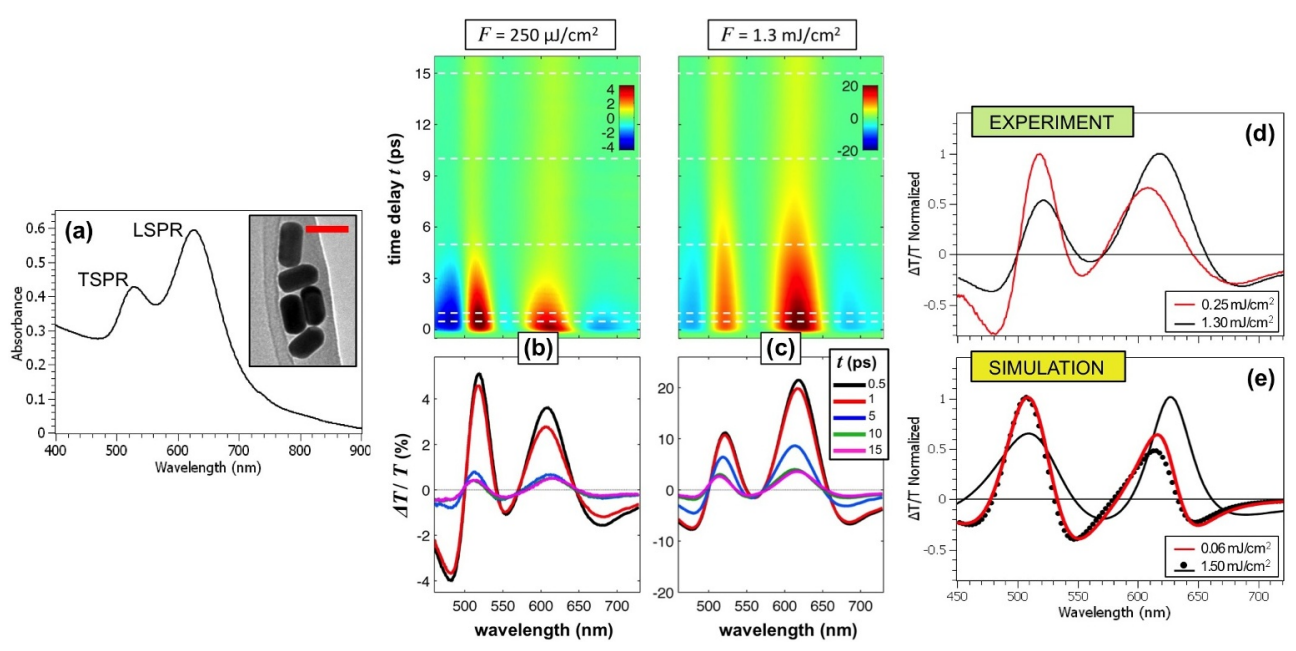

Fig. 1. (a) Steady-state absorbance of the water dispersed GNRs sample. Inset shows a TEM image of the synthesized GNRs, scale bar $50 \mathrm{~nm}$. (b)-(c) Experimental $\Delta \mathrm{T} / \mathrm{T}$ map (top panels) and map crosssections at selected time delays (bottom panels) for (b) low and (c) high pump fluence F. (d)-(e) Experimental and simulated $\Delta \mathrm{T} / \mathrm{T}$ normalized to the higher peak at $1 \mathrm{ps}$ time delay for low (red trace) and high (black traces) pump fluence. In the simulations of panel (e), the black solid curve refers to the actual model with nonlinear Fermi-smearing mechanism and the black dots refer to the linearized model.

The gold nanorods (GNR) samples are synthesized in colloidal solution by the seedless synthesis method [1] in the presence of the cationic surfactant hexadecyltrimethylammonium bromide (CTAB) and resveratrol as a reductant. The size distribution was estimated by transmission electron microscopy (TEM) obtaining an average aspect ratio of 1.9 , with $26 \mathrm{~nm}$ width and $50 \mathrm{~nm}$ length. The GNRs are centrifuged and redispersed in deionized water for the pump-probe measurements.

The optical absorption spectrum of the aqueous solution is shown in Figure 1(a). The two peaks correspond to the surface plasmon resonances (SPR) of the nanorods [2]: the peak at $520 \mathrm{~nm}$ is the transversal SPR (TSPR), whereas the second peak at around $625 \mathrm{~nm}$ is the longitudinal SPR (LSPR).

The $\Delta \mathrm{T} / \mathrm{T}$ map as a function of probe wavelength and pump-probe delay obtained for interband pumping at $400 \mathrm{~nm}$ with $250 \mu \mathrm{J} / \mathrm{cm}^{2}$ fluence is shown in Figure 1(b). The map is characterized by resonant features around the absorption peaks observed in the steady-state spectrum [cf. Figure 1(a)]. These are due to the modification of the dielectric function induced by the pump pulse that in turn affects the spectral location and width of the TSPR and LSPR seen by the probe, in agreement with a vast literature on the subject (see e.g. [3] and references therein). However, when increasing the pump fluence to $1.5 \mathrm{~mJ} / \mathrm{cm}^{2}$ the $\Delta \mathrm{T} / \mathrm{T}$ not only increases and saturates but, most interestingly, modifies its spectral shape on the ultrafast time scale of the first picoseconds, as shown in Figure 1(c). The two plasmonic resonances thus exhibit very different saturation dynamics, as highlighted in the normalized spectra reported in Figure 1(d) for 1 ps time delay: for low fluence [red trace in Figure 1(d)] the TSPR dominates the transient spectrum, whereas for high fluence [black trace in Figure 1(d)] it is the LSPR that prevails. The same behaviour was observed for intraband pumping at $800 \mathrm{~nm}$.

To identify the mechanism underlying such nonlinear dispersive features, we modeled the pump-probe experiments. The GNRs were approximated by nanoellipsoids with an aspect ratio similar to the one measured in the GNR sample, and quasi-static formulas where employed for their absorption and extinction cross-sections computed at an angle 
with respect to the major axis of the structure (so to enable simultaneous excitation of both resonances). The three-temperature model [4] was employed to account for the dynamics of non-thermalized electrons (generated by the pump pulse), hot-electrons thermalized at high temperature (generated by electron-electron scattering) and hot lattice phonons (generated by electron-phonon scattering). The electron (and lattice) heating then induces a transient modification of the dielectric function, which is dominated, on the considered time scale of the very first picoseconds, by a modulation of the interband optical transition of gold, enabled by a broadening of the Fermi-Dirac distribution, the so-called Fermi-smearing mechanism (see e.g. $[4,5,6]$ and references therein).

The simulated transient optical spectra at 1 ps time delay for low and high pump fluence are shown in Figure 1(e). Note the good agreement with the experimental results of Figure 1(d). Interestingly, an attempt to simplify the model by assuming a Fermi smearing linearized with respect to electronic temperature [black dots in Figure 1(e)] - that is the hypothesis originally introduced by Rosei for the perturbative case of low electronic temperatures [7] - fails to reproduce the high fluence spectrum (cf. with black traces). This indicates that the different saturation dynamics observed for the different plasmonic resonances is mostly related to the highly dispersive nonlinearity entailed by the Fermismearing. We estimated that the onset of this nonlinear mechanism takes place when the hot-electrons temperature increases to about $500 \mathrm{~K}$, which is a non-perturbative regime readily achievable in noble metal nanostructures under a moderate pump fluence of several hundred $\mu \mathrm{J} / \mathrm{cm}^{2}$.

In conclusion, we report an experimental and theoretical study on the transient optical response of plasmonic nanostructures beyond the perturbative regime. Gold nanorods dispersed in water have been investigated by ultrafast pump-probe spectroscopy. A quantitative comparison with numerical simulations based on a semi-classical model of hotelectrons and quasi-static formulas for the optical response enabled us to reveal a rather universal mechanism presiding over the saturation dynamics of plasmonic resonances, that is the intrinsic nonlinearity of the Fermi-smearing. quations should be centred and should be numbered with the number on the right-hand side.

We acknowledge financial support from the funding agencies: Fapemig, CNPq, and Capes.

\section{References}

[1] W. Wang, J. Li, S. Lan, L. Rong, Y. Liu, Y. Sheng, H. Zhang and B. Yang, Nanotechnology 27, 165601 (2016).

[2] S. Link, M. B. Mohamed, M. A. El-Sayed, J. Phys. Chem. B 103, 3073 (1999).

[3] X. Wang, Y. Guillet, P. R. Selvakannan, H. Remita, B. Palpant, J. Phys. Chem. C 119, 7416 (2015).

[4] C.-K. Sun, F. Vallée, L. H. Acioli, E. P. Ippen and J. G. Fujimoto, Phys. Rev. B 50, 15337 (1994).

[5] H. Baida, D. Mongin, D. Christofilos, G. Bachelier, A. Crut, P. Maioli, N. Del Fatti, and F. Vallée, Phys. Rev. Lett. 107, 057402 (2011).

[6] M. Zavelani-Rossi, D. Polli, S. Kochtcheev, A.-L. Baudrion, J. Béeal, V. Kumar, E. Molotokaite, M. Marangoni, S. Longhi, G. Cerullo, P.-M. Adam, and G. Della Valle, ACS Photon. 2, 521 (2015).

[7] R. Rosei, Phys. Rev. B 10, 474 (1974) 